\title{
Heavy flavour measurements in the ALICE experiment at the LHC
}

\author{
Giuseppe Eugenio Bruno* for the ALICE Collaboration \\ Dipartimento Interateneo di Fisica “M. Merlin” and Sezione INFN, Bari, Italy \\ E-mail: giuseppe.bruno@ba.infn.it
}

\begin{abstract}
The results on open heavy flavour and charmonium production in pp collisions at the LHC with the ALICE experiment are presented, based on data collected in 2010 at $\sqrt{s}=7 \mathrm{TeV}$. In particular charm production at central rapidity is measured in a number of exclusive decay channels into charged hadrons, e.g. $\mathrm{D}^{0} \rightarrow \mathrm{K}^{-} \pi^{+}, \mathrm{D}^{+} \rightarrow \mathrm{K}^{-} \pi^{+} \pi^{+}$and $\mathrm{D}^{*+} \rightarrow \mathrm{D}^{0} \pi^{+}$. Beauty and charm production can be studied at central and forward rapidity through the inclusive channels decaying in single electron and muon, respectively; $\mathrm{J} / \psi$ production at central and forward rapidities has been measured in the dielectron and dimuon channels, respectively. Finally, perspectives on heavy flavour measurements in $\mathrm{Pb}-\mathrm{Pb}$ collisions are discussed.
\end{abstract}

The 13th International Conference on B-Physics at Hadron Machines - Beauty2011, April 04-08, 2011

Amsterdam, The Netherlands

${ }^{*}$ Speaker. 


\section{Introduction}

The ALICE detector is very different in both design and purpose from the other experiments at the CERN Large Hadron Collider (LHC). Its main aim is the study of matter under extreme conditions of temperature and pressure, i.e. the Quark-Gluon Plasma (QGP), in collisions between heavy ions. With a present energy up to almost 14 times higher than that of RHIC, the previous energy frontier machine for heavy-ion collisions at BNL, we expect a different type of QGP, e.g. in terms of initial temperature, lifetime and system volume, and abundances of hard signals like jets and heavy quarks which serve as probes to study the QGP properties. Data taking with protonproton collisions is important for ALICE, primarily to collect the reference data for the heavy-ion programme. Therefore, our goal in 2010 was to collect about $10^{9}$ Minimum-Bias (MB) pp collisions, which provide sufficient statistics for comparison with the first heavy-ion run accomplished in November 2010.

Heavy-flavour hadrons are regarded as effective probes of the conditions of the system produced in nucleus-nucleus collisions. In particular, open charm and beauty hadrons would be sensitive to the energy density, through the mechanism of in-medium energy loss of heavy quarks; quarkonium states would be sensitive to the initial temperature of the system, through their dissociation due to colour screening; initially uncorrelated charm and anti-charm quarks, abundantly produced in the initial stage of the collision, may recombine and yield an increase in the number of observed charmonium particles.

Heavy-quark production measurements in proton-proton collisions at LHC energies are interesting per se, as a test of perturbative QCD (pQCD) in a new energy domain. State-of-the-art implementations of $\mathrm{pQCD}$ calculations describe well the beauty production cross section measured in pp collisions at a centre of mass energy $\sqrt{s}=1.96 \mathrm{TeV}$ at the Tevatron [1]. Also the production of charm hadrons (D mesons) is reproduced within the theoretical uncertainties of the pQCD calculation [2]. However, in this case the comparison suggests that charm production is slightly underestimated in the calculations, as observed also in pp collisions at RHIC at the much lower $\sqrt{s}$ of $0.2 \mathrm{TeV}$ [3, 4]. Moving from open to hidden heavy flavour production, the hadroproduction of quarkonium states is a process where QCD is involved in both perturbative and non-perturbative aspects. Various models [5] have been proposed to describe the results obtained at the Tevatron [6, 7], but they fail to reproduce simultaneously the production cross sections, the transverse momentum distributions, and the measured polarization, as well as their dependence on rapidity.

\section{Detector}

The ALICE detector consists of a central part, which measures hadrons, electrons and photons, and a forward spectrometer to measure muons. The central part, which covers the pseudorapidity range of $|\eta|<0.9$ over the full azimuth, is embedded in a large solenoidal magnet supplying a field of $0.5 \mathrm{~T}$. This part consists of: an Inner Tracking System (ITS) of high-resolution silicon detectors; a cylindrical Time-Projection Chamber (TPC); three particle-identification arrays of Time-OfFlight (TOF), Transition-Radiation-Detector (TRD), and Čerenkov-ring-imaging (called HMPID) counters; two electromagnetic calorimeters (high resolution PHOS and large acceptance EMCAL). The forward muon spectrometer covering $-4<\eta<-2.5$ consists of a complex arrangement of 
absorbers, a large dipole magnet with a field integral of $3 \mathrm{Tm}$, and ten tracking stations and two trigger chamber stations. Several smaller detectors for triggering and multiplicity measurements (VZERO, T0, FMD, PMD, ZDC) are located at small angles. The main design features include: a robust but limited-pseudorapidity acceptance tracking system, designed with redundancy to cope with the very high particle density in nuclear collisions; a minimum of material in the sensitive tracking volume (10\% of radiation length between interaction point and outer radius of the TPC) to reduce multiple scattering; several detector subsystems dedicated to particle identification over a large range in momentum. The layout of the ALICE detector and its eighteen different subsystems are described in detail in [8].

\section{Exclusive hadronic decay channels of charmed hadrons}
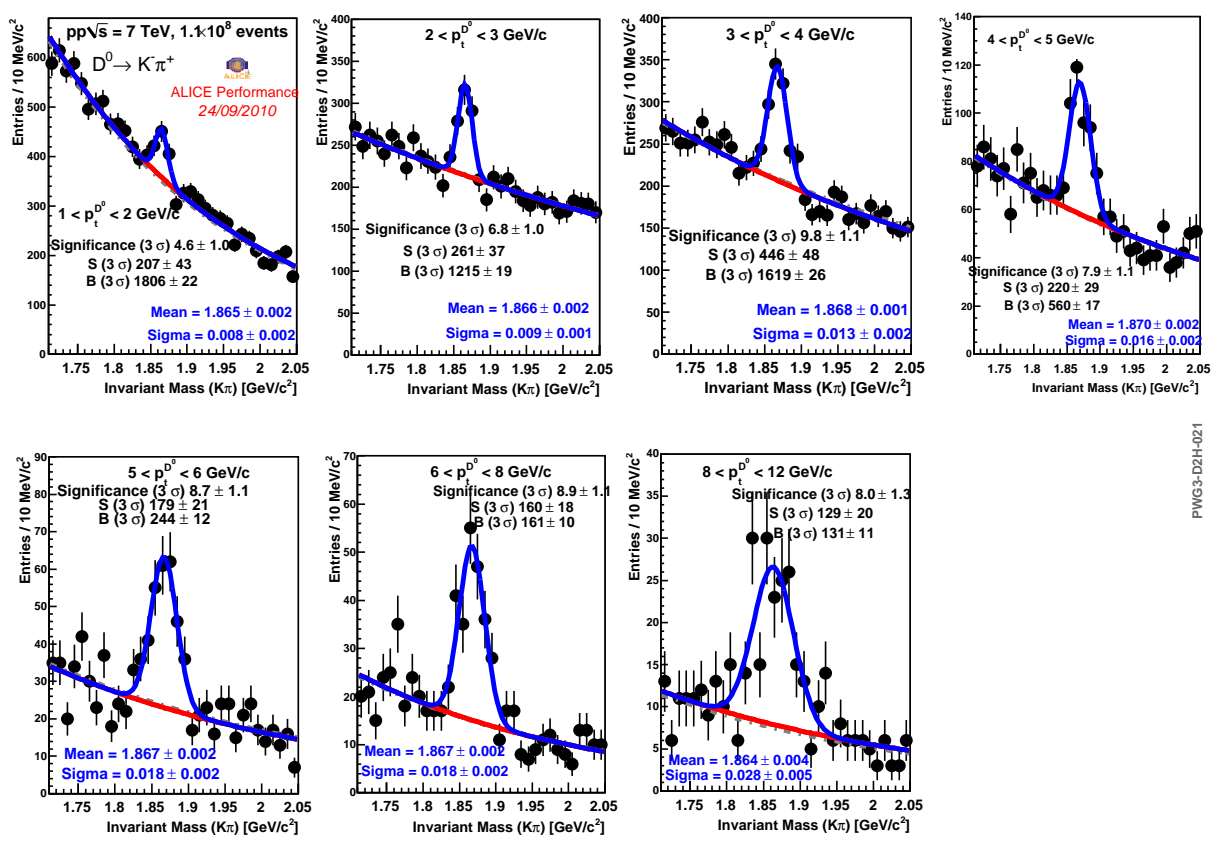

Figure 1: Invariant mass distributions for opposite sign charged $\mathrm{K}$ and $\pi$ pairs after kinematical and topological cuts of $\mathrm{D}^{0}$ candidates in seven $p_{\mathrm{T}}$ bins for a sample of $1.1 \cdot 10^{8} \mathrm{MB}$ events. Superimposed are the results of the fits (see text for details).

ALICE can study charmed hadron production at central rapidity down to very low momenta using various exclusive decay modes into charged hadrons, namely $\mathrm{D}^{0} \rightarrow \mathrm{K}^{-} \pi^{+}, \mathrm{D}^{+} \rightarrow \mathrm{K}^{-} \pi^{+} \pi^{+}$, $\mathrm{D}_{\mathrm{s}}^{+} \rightarrow \mathrm{K}^{+} \mathrm{K}^{-} \pi^{+}, \mathrm{D}^{*+} \rightarrow \mathrm{D}^{0} \pi^{+}, \mathrm{D}^{0} \rightarrow \mathrm{K}^{-} \pi^{+} \pi^{-} \pi^{+}$and $\Lambda_{\mathrm{c}}^{+} \rightarrow \mathrm{pK}^{-} \pi^{+}$, and their charge conjugates for the corresponding anti-particles. Kinematical and topological cuts are applied to drastically reduce the combinatorial background and obtain invariant mass ditributions with good signal over background ratios $(S / B)$ and significances $(S / \sqrt{S+B})$ even at transverse momenta down to 1 $\mathrm{GeV} / c$, thanks to the use of particle identification and the excellent performance of the ITS vertex detector, which provides an impact-parameter resolution of around $75 \mu \mathrm{m}$ at $p_{\mathrm{T}}=1 \mathrm{GeV} / c$. As an example, in Fig. 1 the invariant mass distributions of the $\mathrm{D}^{0}$ candidates into opposite sign charged 

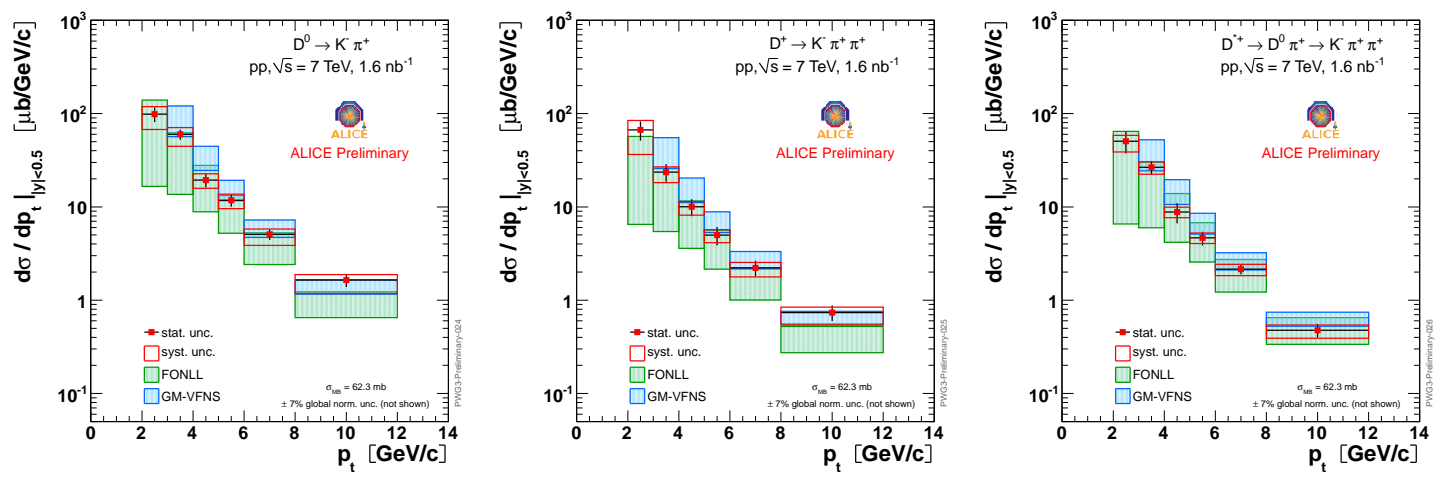

Figure 2: Preliminary $p_{\mathrm{T}}$ differential cross section for $\mathrm{D}^{0}$ (left panel), $\mathrm{D}^{+}$(central panel) and $\mathrm{D}^{*}$ (right panel) compared with FONLL [9] and GM-VFNS [10] theoretical predictions.

$\mathrm{K}$ and $\pi$ pairs are shown for different $p_{\mathrm{T}}$ bins. Superimposed are the results of best fits using Gaussian distributions to describe the signal and exponentials for the background. The raw yields, extracted from the invariant mass analyses, have been corrected for the acceptance of the apparatus and for PID, selection and reconstruction efficiencies, using a detailed detector simulation. The contamination of D mesons from B meson decays is currently estimated to be about 10-15\%, using the ratio of beauty over charm production cross sections predicted by the FONLL (fixed-order nextto-leading log) calculation [9] and the detector simulation, and it is subtracted from the measured raw $p_{\mathrm{T}}$ spectrum, before applying the efficiency corrections (calculated for prompt $\mathrm{D}$ mesons). A data driven method to subtract this contamination will be used with the full 2010 statistics. The $p_{\mathrm{T}}$ differential cross sections for $\mathrm{D}^{0}, \mathrm{D}^{+}$and $\mathrm{D}^{*}$ in pp collisions at $\sqrt{s}=7 \mathrm{TeV}$ are shown in Fig. 2 for a data sample corresponding to an integrated luminosity $L_{\text {int }}=1.6 \mathrm{nb}^{-1}$ (about $25 \%$ of the 2010 data sample). The measured differential prompt $\mathrm{D}$ hadron cross-sections are well described by FONLL [9] and GM-VFNS [10] pQCD calculations.

\section{Leptons from heavy-flavour decays}

The production of open charm and beauty can be studied by detecting the semi-leptonic decays of $\mathrm{D}$ and $\mathrm{B}$ mesons which each have a branching ratio of about $10 \%$ [11].

Muons: The extraction of the heavy-flavour contribution from the single muon spectra requires the subtraction of three main sources of background: a) muons from the decay-in-flight of light hadrons (decay muons); b) muons from the decay of hadrons produced in the interaction with the front absorber (secondary muons); c) punch-through hadrons. The last contribution can be efficiently rejected by requiring the matching of the reconstructed tracks with the tracks in the trigger system. Due to the lower mass of the parent particles, the background muons have a softer transverse momentum than the heavy-flavour muons, and dominate the low- $p_{\mathrm{T}}$ region. The analysis focuses on the region $2<p_{\mathrm{T}}<6.5 \mathrm{GeV} / c$, the upper limit being determined by the $p_{\mathrm{T}}$ resolution of the spectrometer with the initial, only partial, alignment. Simulation studies indicate that, in this momentum range, the contribution of secondary muons is small (about 3\%). Here the main source of background consists of decay muons (about 25\%), which have been subtracted by means 
of Monte Carlo simulations. After background subtraction, the muon $p_{\mathrm{T}}$ spectrum is corrected for efficiency, using the Monte Carlo detector simulation. The charm and beauty decay muon cross section $\mathrm{d} \sigma_{\mu} / \mathrm{d} p_{\mathrm{T}}$ in the range $2<p_{\mathrm{T}}<6.5 \mathrm{GeV} / c$ and $-4<\eta<-2.5$ is presented in Fig. 3. The corresponding FONLL pQCD calculation [9] agrees with the data within uncertainties.

The next step in this analysis is the extension of the high- $p_{\mathrm{T}}$ reach up to about $20 \mathrm{GeV} / c$, using data collected since summer 2010 that have been reconstructed with improved alignment corrections of the tracking chambers. The inclusive single-muon cross section will be dominated by beauty decays in the range $10<p_{\mathrm{T}}<20 \mathrm{GeV} / c$, therefore this measurement will constitute the reference for the study of $\mathrm{b}$ quark quenching at forward rapidity in $\mathrm{Pb}-\mathrm{Pb}$ collisions.

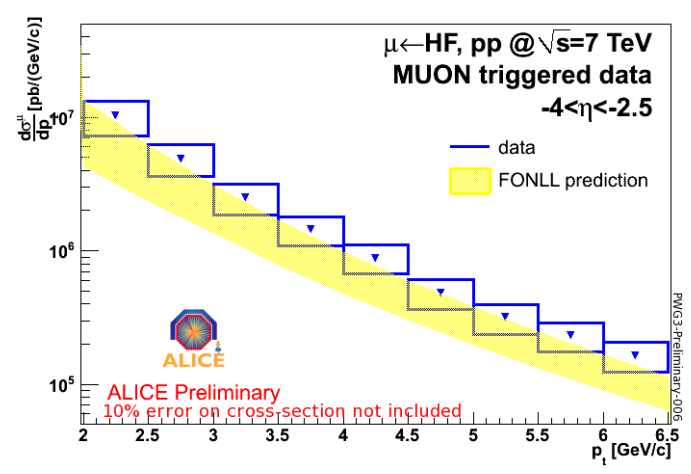

Figure 3: Differential transverse momentum cross-section of muons coming from semi-muonic decay of heavy-flavour hadrons $\mathrm{d} \sigma_{\mu} / \mathrm{d} p_{\mathrm{T}}$ measured in the pseudorapidity range $-4<\eta<-2.5$ in comparison with the FONLL predictions [9]. The systematic errors are shown with boxes and do not include an additional $10 \%$ error on the MB cross-section, while the statistical errors are small (hidden by the markers). Results have been obtained from a sample of events corresponding to $L_{\mathrm{int}}=3.49 \mathrm{nb}^{-1}$.

Electrons: The open heavy-flavour production cross section at mid-rapidity can be studied through the measurement of single electrons. Those electrons are identified on a statistical basis by subtracting the "cocktail" of background electrons from the inclusive electron spectrum. This background arises mainly from electrons from $\gamma$ conversion in the detector material and $\pi^{0}$ Dalitz decays. For $p_{\mathrm{T}}$ up to few $\mathrm{GeV} / c$ this cocktail can be determined precisely by means of the measured hadron cross sections, in particular that of the $\pi^{0}$. The basis of this measurement is, clearly, a robust electron identification. This initial analysis focuses on using the TPC and TOF detectors. The TOF signal allows rejection of most of the kaons and protons, that cross the electron $\mathrm{d} E / \mathrm{d} x$ band in the TPC at $p \approx 0.5$ and $1.2 \mathrm{GeV} / c$, respectively. Then, a cut on the TPC $\mathrm{d} E / \mathrm{d} x$ allows separation of the electrons from the pions up to about $4 \mathrm{GeV} / c$. The residual pion contamination is lower than $15 \%$ in this momentum range. The corrected inclusive electron spectrum is shown in Fig. 4 (left panel) together with the cocktail of background electrons. Figure 4 (right panel) illustrates the cross section of single electrons coming from open heavy flavour hadrons, which has a total systematic uncertainty of $16-20 \%$ ( $p_{\mathrm{T}}$ dependent) plus $7 \%$ for the normalization. The data are well described by FONLL calculations [9] within errors. Moreover, the low $p_{\mathrm{T}}$ single electron spectrum agrees with the electron yield from $\mathrm{D}$ meson decays computed from the measured $\mathrm{D}$ meson cross sections (discussed above). The $p_{\mathrm{T}}$ reach will be extended with the TRD and EMCAL detectors in the near future and electrons from beauty decays will be identified through displaced vertices. 

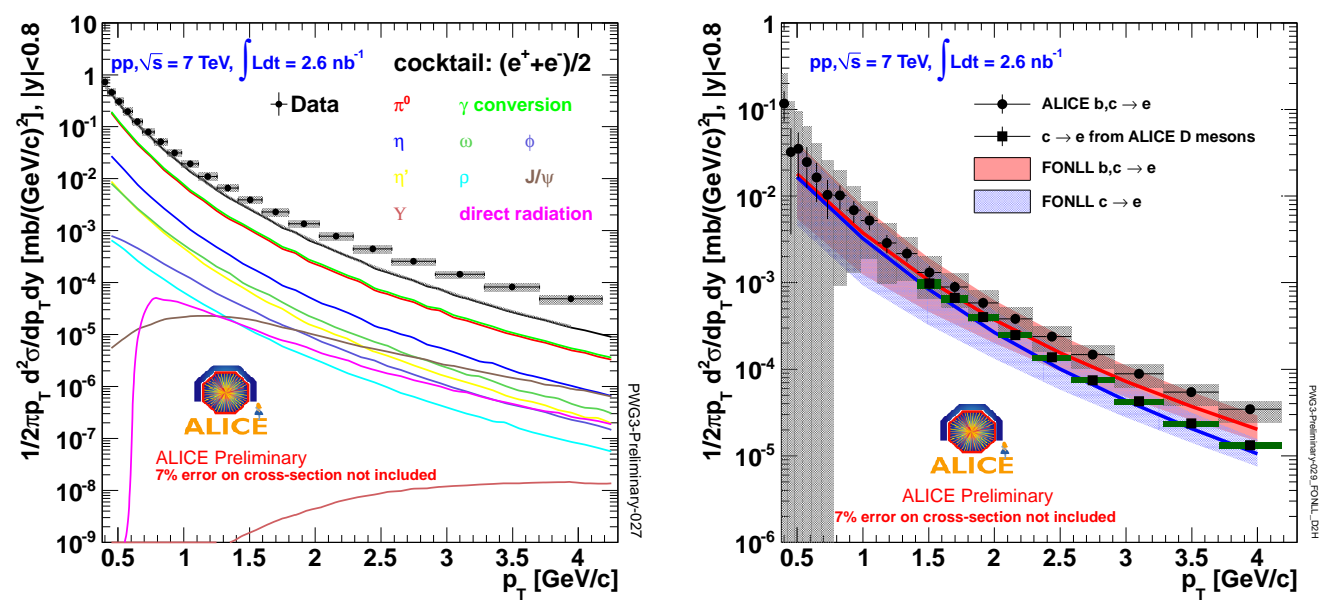

Figure 4: Left: the inclusive electron spectrum as a function of transverse momentum measured in ALICE at mid-rapidity $(|y|<0.8)$. Superimposed is the electron cocktail which describes the main background sources to the electrons from heavy flavour decay. Right: differential transverse momentum cross-section of electrons coming from semi-electronic decays of heavy-flavour hadrons (charm and beauty, closed circles, with the grey regions indicating the systematic uncertanties), and from semi-electronic decays of charm particles only (closed squares, with the green boxes showing the systematic uncertanties). These are obtained, respectively, as the difference between the inclusive spectrum and the electron cocktail and from the measured charm meson cross-sections (see text for details). Predictions from FONLL cross-sections [9] are also superimposed for the electrons coming from both charm and beauty hadrons (red line) and from charm only (blue line), with their uncertainties drawn as red and blue bands, respectively.

\section{Charmonium measurements}

Heavy quarkonia states are measured in ALICE at central and forward rapidities through their $\mathrm{e}^{+} \mathrm{e}^{-}$and $\mu^{+} \mu^{-}$decays, respectively. The rapidity and transverse momentum dependence of inclusive $\mathrm{J} / \psi$ production has been measured in pp collisions at $\sqrt{s}=7 \mathrm{TeV}$, based on data samples corresponding to integrated luminosities $L_{\mathrm{int}}=3.9 \mathrm{nb}^{-1}$ and $L_{\mathrm{int}}=15.6 \mathrm{nb}^{-1}$ in the dielectron and dimuon channels, respectively [12]. The results are presented in Fig. 5 for the $p_{\mathrm{T}}$-differential cross section $\mathrm{d}^{2} \sigma_{\mathrm{J} / \psi} / \mathrm{d} p_{\mathrm{T}} \mathrm{d} y$ and $\mathrm{d} \sigma_{\mathrm{J} / \psi} / \mathrm{d} y\left(p_{\mathrm{T}}>0\right)$. For the rapidity distribution, the values obtained in the forward region are reflected with respect to $y=0$. The results are compared with those by the CMS [13], LHCb [14] and ATLAS [15] collaborations. Taking together the results from the dimuon and dielectron channels, the ALICE measurement of the inclusive $\mathrm{J} / \psi$ production cross section is particularly relevant in the context of charmonium studies at the LHC, for its coverage of both central and forward rapidities and for the lowest $p_{\mathrm{T}}$ reach at $y=0$. The measured inclusive production is a superposition of a direct component and of $\mathrm{J} / \psi$ coming from the radiative decay of higher-mass charmonium states. In addition to this "prompt" production, decays of particles with beauty are also known to give a sizeable contribution to the observed $\mathrm{J} / \psi$ yield. With future highstatistics data samples, the ALICE experiment will identify, at central rapidity, $\mathrm{J} / \psi$ from b-decays, via the measurement of the pseudo-proper decay length distributions [16], and will reconstruct the $\chi_{\mathrm{c}} \rightarrow \mathrm{J} / \psi+\gamma$ decay [17]. At forward rapidity, the contribution from b-decays will be estimated 
from the beauty cross section measurement carried out in the semi-leptonic decay channel. Finally, the total collected statistics at $\sqrt{s}=7 \mathrm{TeV}$ allows the determination of the full angular distribution of the $\mathrm{J} / \psi$ decay muons; the expected errors on the polarization parameters for an analysis in the range $3<p_{\mathrm{T}}<8 \mathrm{GeV} / c$ are small enough to discriminate between different theoretical predictions (e.g., smaller than 0.15 for the $\lambda_{\theta}$ parameter, whose definition can be found, e.g., in [18]).
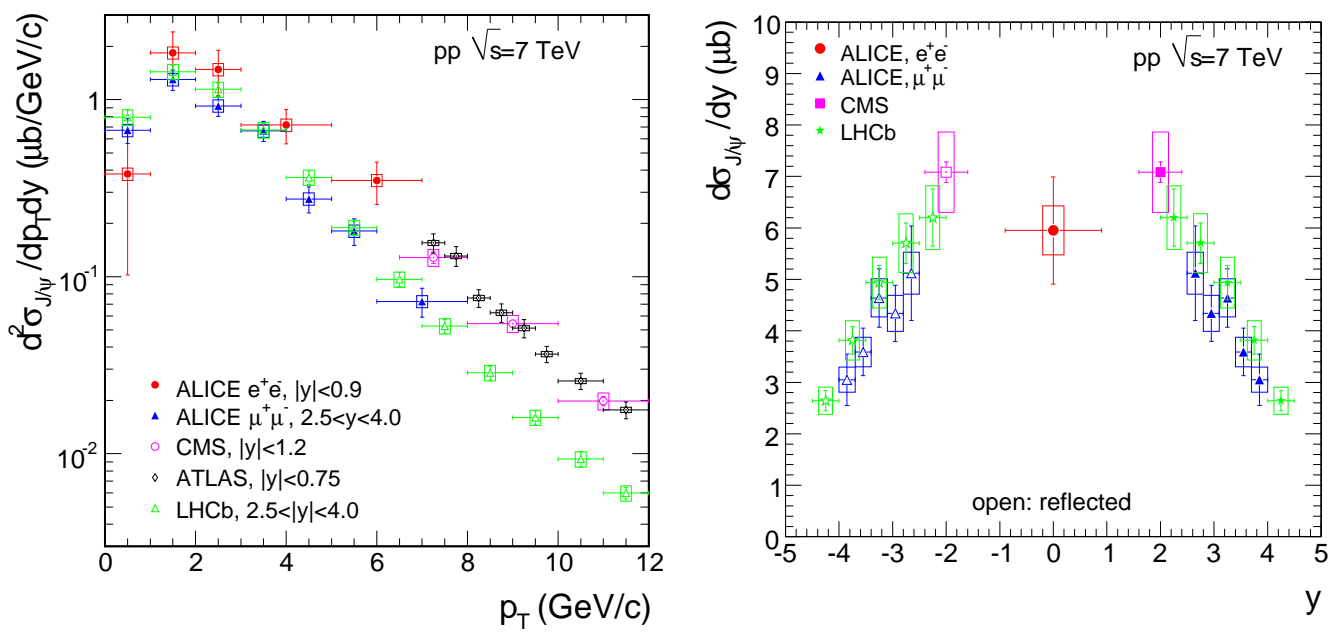

Figure 5: Left: $\mathrm{d}^{2} \sigma_{\mathrm{J} / \psi} / \mathrm{d} p_{\mathrm{T}} \mathrm{d} y$ for the mid-rapidity range and for the forward rapidity data [12], compared with results from the other LHC experiments $[13,14,15]$, obtained in similar rapidity ranges. The error bars represent the quadratic sum of the statistical and systematic errors, while the systematic uncertainties on luminosity are shown as boxes. Right: $\mathrm{d} \sigma_{\mathrm{J} / \psi} / \mathrm{d} y$ [12], compared with results from the other LHC experiments with coverage down to $p_{\mathrm{T}}=0[13,14,15]$. The error bars represent the quadratic sum of the statistical and systematic errors, while the systematic uncertainties on luminosity are shown as boxes.

\section{Outlook to $\mathrm{Pb}-\mathrm{Pb}$}

In the first heavy ion run of the LHC, which took place in November 2010, ALICE has collected data using slightly different MB triggers which selected a data sample corresponding to the most central 97-99\% (the exact value depending on the particular trigger) of the inelastic $\mathrm{Pb}-\mathrm{Pb}$ cross-section at the center of mass energy per nucleon pair $\sqrt{s_{\mathrm{NN}}}=2.76 \mathrm{TeV}$; the final sample which can be used for physics studies amounts to about 30 million such collisions. A number of physics results have been obtained, based on a fraction of these data, which characterizes the global properties of the system created in $\mathrm{Pb}-\mathrm{Pb}$ collisions at this energy [19, 20, 21, 22]. In particular, the observed suppression of charged particle production at large transverse momentum in central collisions [23] is stronger than that observed at lower collision energies, indicating that a very dense medium is formed in central $\mathrm{Pb}-\mathrm{Pb}$ collisions at the LHC. Similar measurements performed using heavy flavour probes (e.g., open charm and charmonium) would be fundamental to address the mechanism of in-medium energy loss of heavy quarks, to estabilish the initial temperature of the system, and to understand the hadronization process in heavy ion collisions. In Fig. 6 the invariant mass distributions for $\mathrm{D}^{0} \rightarrow \mathrm{K} \pi, \mathrm{D}^{+} \rightarrow \mathrm{K} \pi \pi$ and $\mathrm{J} / \psi \rightarrow \mu^{+} \mu^{-}$candidates are shown after kinemati- 
$\mathrm{cal}$ and topological cuts for most central $\mathrm{Pb}-\mathrm{Pb}$ collisions. These data will enable the measurements discussed above.
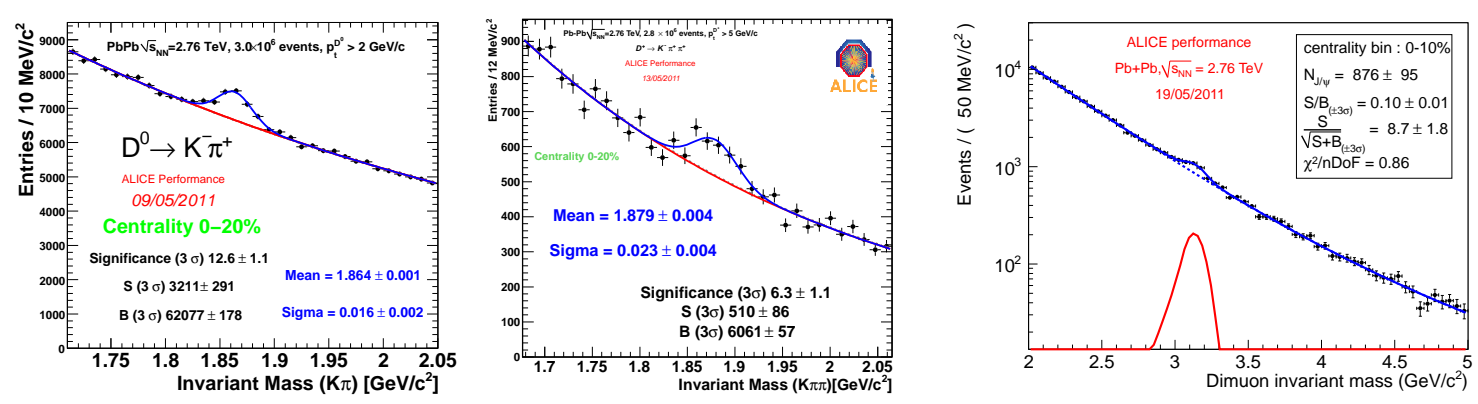

Figure 6: Invariant mass distributions for charged $\mathrm{K} \pi$ pairs (left panel) and $\mathrm{K} \pi \pi$ triplets (central panel) in the rapidity range $|y|<0.8$ for the most central $20 \%$ of the inelastic $\mathrm{Pb}-\mathrm{Pb}$ cross section at $\sqrt{s_{\mathrm{NN}}}=2.76$ TeV. Right panel: invariant mass distribution of $\mu^{+} \mu^{-}$candidates in the rapidity range $-4<y<-2.5$ for events corresponding to the most central $10 \%$ of the inelastic $\mathrm{Pb}-\mathrm{Pb}$ cross section at $\sqrt{s_{\mathrm{NN}}}=2.76 \mathrm{TeV}$.

\section{References}

[1] M. Cacciari et al., JHEP 0407 (2007) 033.

[2] D. Acosta et al. (CDF Collaboration), Phys. Rev. Lett. 91 (2003) 241804.

[3] A. Adare et al. (PHENIX Collaboration), Phys. Rev. Lett. 97 (2006) 252002.

[4] W. Xie et al. (STAR Collaboration), Nucl. Phys. A 855 (2011) 108-115.

[5] N. Brambilla et al. (Quarkonium Working Group), arXiv:1010.5827v1[hep-ph]

[6] D. Acosta et al. (CDF Collaboration), Phys. Rev. D71 (2005) 032001.

A. Abulencia et al. (CDF Collaboration), Phys. Rev. Lett. 99 (2007) 132001.

[7] S. Abachi et al. (D0 Collaboration), Phys. Lett. B370 (1996) 239.

B. Abbott et al. (D0 Collaboration), Phys. Rev. Lett. 82 (1999) 35.

[8] K. Aamodt et al. (ALICE Collaboration), J. Instrum. 3 (2008) S08002

[9] M. Cacciari, M. Greco, P. Nason, JHEP 9805 (1998) 007; private communication.

[10] B.A. Kniehl et al., Phys. Rev. Lett. 96 (2006) 012001; private communication.

[11] K. Nakamura et al. (Particle Data Group), J. Phys. G 37 (2010) 075021.

[12] K. Aamodt et al. (ALICE Collaboration), arXiv:1105.0380, submitted to Phys. Lett. B.

[13] V. Khachatryan et al. (CMS Collaboration), Eur. Phys. J. C 71 (2011) 1575.

[14] R. Aaij et al.(LHCb Collaboration), Eur. Phys. J. C 71 (2011) 1645.

[15] G. Aad et al. (ATLAS Collaboration), arXiv:1104.3038, submitted to Nucl. Phys. B.

[16] G.E. Bruno et al. (ALICE Collaboration), PoS (ICHEP2010) 194.

[17] P. Gonzalez et al. (ALICE Collaboration), Eur. Phys. J. C 61 (2009) 899.

[18] P. Faccioli et al., Eur. Phys. J. C 69 (2010) 657-673. 
[19] K. Aamodt et al. (ALICE Collaboration), Phys. Rev. Lett. 105 (2010) 252301.

[20] K. Aamodt et al. (ALICE Collaboration), Phys. Rev. Lett. 105 (2010) 252302.

[21] K. Aamodt et al. (ALICE Collaboration), Phys. Lett. B 696 (2011) 328-337.

[22] K. Aamodt et al. (ALICE Collaboration), Phys. Rev. Lett. 106 (2011) 032301.

[23] K. Aamodt et al. (ALICE Collaboration), Phys. Lett. B 696 (2011) 30-39. 